\title{
Hot gas filtration using a moving bed heat exchanger-filter (MHEF)
}

\author{
V. Henriquez, A. Macias-Machin * \\ Grupo Energy' and Environment (EMA), E.T.S.I.I. de Las Palmas, Edificio de Ingenierias, Campus Universitario de Tafira, ULPGC, \\ Las Palmas de G.C., E-35017, Spain \\ Received 18 December 1996; accepted 2 April 1997
}

\begin{abstract}
A study was conducted on the use of a Moving Bcd Heat Exchangcr-Filter (MHEF) for removing fine dust particles from gascs. The influence of a number of variables was examiñed, including gas velocities, solid velocities, gas temperatures and dust sizes. The collection efficiency was found to decrease with increasing temperature; the total collection efficiency decreases strongly when the solid velocity increases. A stable numerical model for filtration and heat exchange was developed that predicts the two dimensional transient response of both solid and fluid phases. The numerical model incorporates variation in void fraction, velocities and transport coefficient due to combined processes of filtration and heat exchange. (C) 1997 Elsevier Science S.A.
\end{abstract}

Keywords: Gas filtration; Granular filter; Heal exchanger

\section{Introduction}

Granular filtration through fixed, moving and fluidized bed filters has recently attracted our attention due to the fact that this filtration can be used for high temperatures and high pressure. These conditions can be found in the clean-up of gases from energy production processes such as coal gasification or fluidized bed combustion [1].

In these types of granular filters, the polluting gas gets into contact with the granular filter (sand, gravel, metallic particles, etc.) taking place the filtration process of polluting gases, and where the granular filter holds the particulate solids which carry on the polluting gases.

In addition to this, polluting hot gases are found at a middle-high temperature. Depending on the flow of gases, energy can be recovered in this way, therefore, we can save energy. This saving can be obtained by means of solid-gas exchangers or heat regenerators.

$\mathrm{Up}$ to now, a series of equipment has been developed related to the fluid-particle heat transfer [2-5]. In many of these designs, heat exchange takes place between

\footnotetext{
* Corresponding author. Fax: + 3428 610029; e-mail: anacias@cicei.ulpgc.es
}

particles which fall and hot gases which go up or use a packing of large diameter placed at the top of the exchanger to preheat particles. In all the previous cases, a heat exchanger which filters particulate solids simultaneously and a granular filter which recovers energy are not mentioned. This is due to the fact that both polluting hot gas filters and solid-gas heat recuperators have generally been studied, designed and developed separately.

At the present time, a series of equipment that combine basic operations of filtration and heat exchange simultaneously are being developed. Among these devices we have the moving bed heat exchanger-filter (MIIEF) [6].

This device allows us to eliminate particulate solids of polluting hot gases and recover the heat of these gases which have been exchanged with the granular material of the MHEF. In Fig. 1, we can observe in a schematic way the new design of the heat exchangerfilter (MHEF) that uses a magnetic recirculation of solid, so that the device can work continually.

This device consists of the following parts:

1. Upper part: In this part, filtration and heat-exchange operations take place.

2. Device of filter cleanness: In this part, particulate solids are separated from the grain of the filter. 
3. Lower part: In this part, we can see the heat recovery of the gas which goes in crossflow and the granular material that goes down.

4. Magnetic Recirculation of Solids: This part permits the transport of granular material to the upper part of the MHEF so that the device can work continually.

The working process of the different parts as well as details about the different basic operations that take part in the device are given by Jaraiz et al. [6].

Finally, this new device has not been built in a large-scale. At the moment, it is being developed at pilot plant levels. Due to this fact, there are few scientific works about it [7-9]. Therefore, we have tried to develop this new MHEF in this study, focusing our attention on the upper part (A) of the device where

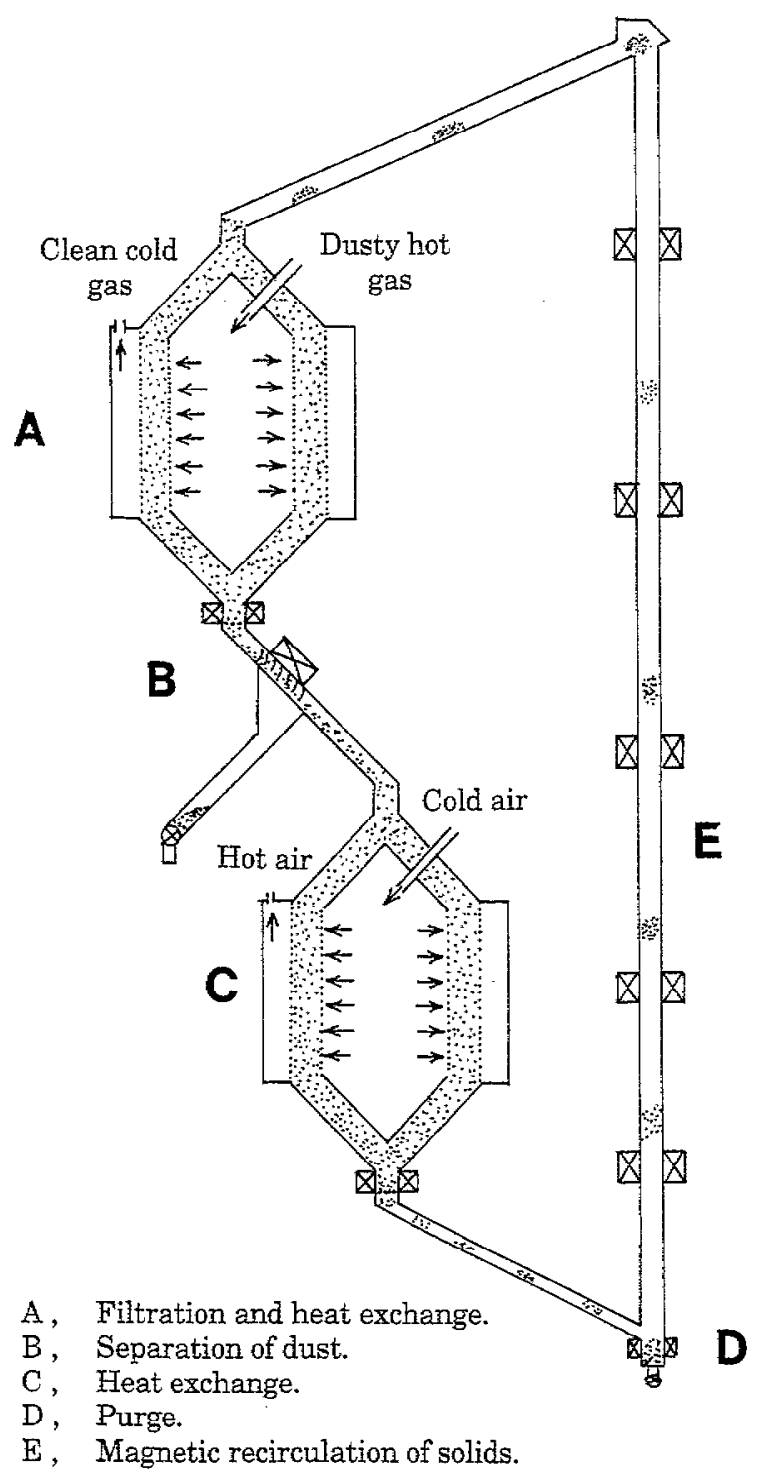

Fig, 1. Schematic diagram of a crossfiow moving bed heat exchangerfilter. filtration and heat-exchange processes take place simultaneously (Fig. 1).

The purpose of the present work was to conduct a systematic experimental study of gas filtration and heat exchange in the upper part of the MHEF. In Fig. 2, we have the schematic diagram of the experimental apparatus used in this work where we have investigated the effect of various operating variables, such as solid velocity, gas velocity, gas and solid temperature. And we have calculated the total collection efficiency and the thermal efficiency of the MHEF.

\section{Experimental work}

The apparatus used in the experimental work is shown in Fig. 2 and consists of the following:

\subsection{Dust generating system}

A fluidized bed dust generator was used to prepare the test suspensions used in the experiment. The fluidized bed material used was a mixture of large sand particles $(500 \mu \mathrm{m})$ and $10 \%$ of fine particles $\left(\mathrm{CaCO}_{3}\right.$, $\left.d_{\mathrm{p}}=12-20 \mu \mathrm{m}\right)$. The fluidized bed had $9.4 \mathrm{~cm}$ I.D. and $1.78 \mathrm{~m}$ height (from distributor) consisting of a mesh nylon screen between perforated stainless steel plates. Dust stability with regard to time has been obtained by means of the installation of a decanting-mixing chamber at the outlet of the fluidized bed. It can be observed that the dust concentration is constant from a moment higher than $5 \min [9]$.

The details of the dust particle size distribution can be seen in the study by Henriquez [10]. We used the following diameter of the dust particles in the experiments:

1. Mean diameter of the dust $\left(\mathrm{CaCO}_{3}\right)$ in the fluidized bed $(70-75 \mu \mathrm{m})$.

2. Mean diameter of the dust $\left(\mathrm{CaCO}_{3}\right)$ before the decanting-mixing chamber $(45-55 \mu \mathrm{m})$.

3. Mean diameter of the dust $\left(\mathrm{CaCO}_{3}\right)$ after the decanting-mixing chamber $(12-20 \mu \mathrm{m})$.

The decanting-mixing chamber has a nozzle located at the lower part, which supplies air at different temperatures from a blower which is connected to a heat exchanger.

\subsection{Moving bed heat exchanger-filter}

The moving bed was made of a steel sheet with three sections (entry section, filter section and discharge section) flanged together. The entry section began with a hopper which had a cross section of $50 \mathrm{~cm}$ by $50 \mathrm{~cm}$ and a height of $50 \mathrm{~cm}$. The discharge section was $50 \mathrm{~cm}$ high with a section similar to the entry section. The filter section was composed of a solid flow duct $(50 \mathrm{~cm}$ 


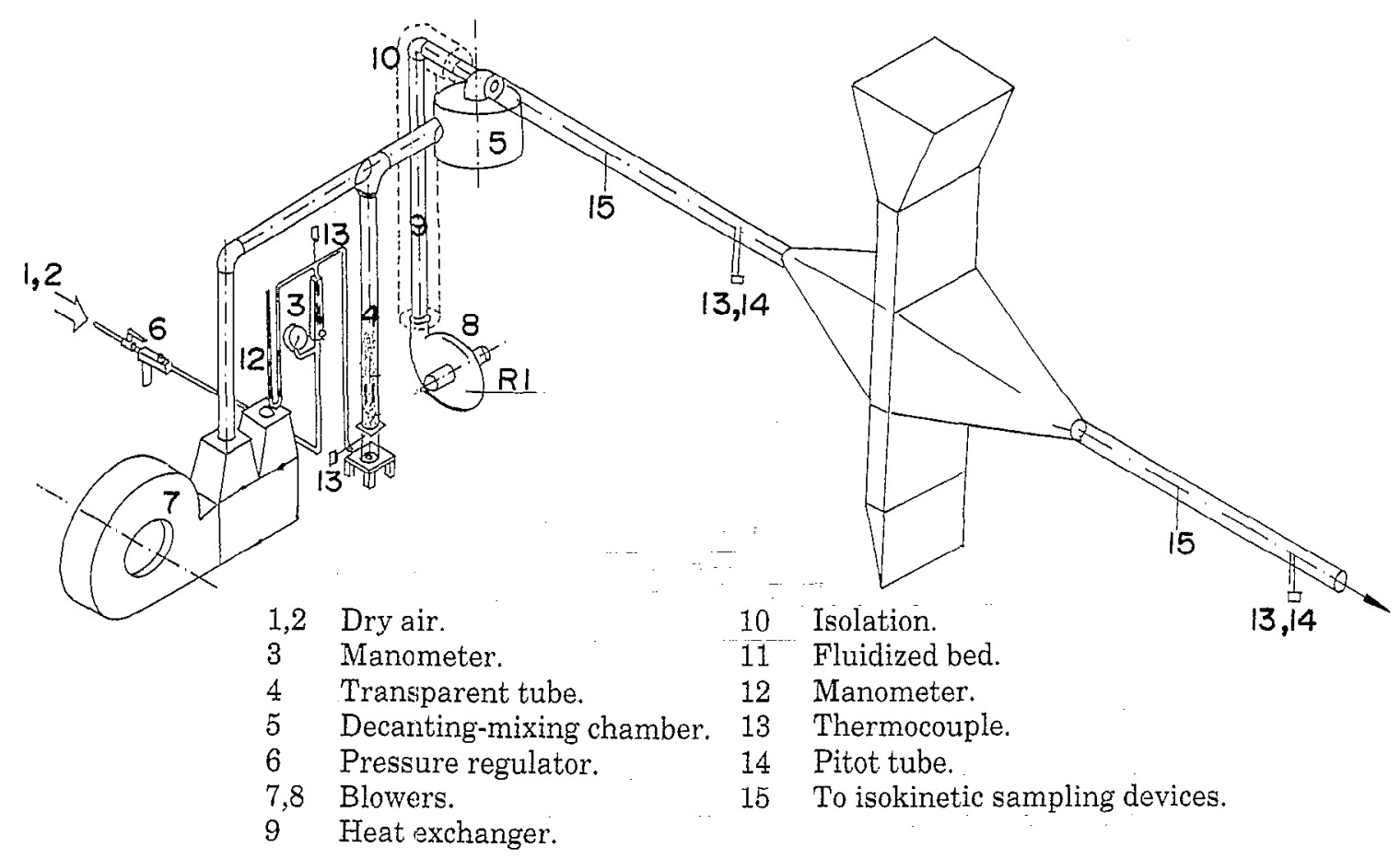

Fig. 2. Schematic diagram of the experimental apparatus.

high) and a gas flow duct $(0.15 \mathrm{~cm}$ long). Both ducts had an identical cross section $(50 \mathrm{~cm} \times 50 \mathrm{~cm})$.

Downward movement of the filter grain was maintained at a velocity of 1 to $4 \mathrm{~cm} \mathrm{~min}^{-1}$ using different openings of the discharge section.

\subsection{Sampling system}

The influent dust concentrations were determined by weighing particles collected by a Watman filter paper No. 1. The effluent dust concentrations were determined by analysing Calcium using an Atomic Spectrophotometer GBC/904. The method used to determine the effluent dust concentration with an Atomic Spectrophotometer consists of the intake of the sample which is going to be analysed in a gaseous current (acetylene and nitrogen dioxide, $\mathrm{N}_{2} \mathrm{O}$ ) and where the metal in solution $(\mathrm{Ca})$ is pulled by this mixture of gases taking place an absorption of the energy corresponding to its concentration in a determined wavelength.

In order to get the concentration of $\mathrm{Ca}$ a calibrated curve was established [10] after passing some samples of a known concentration through the Atomic Spectrophotometer. The samples obtained by a Watman filter paper No. 1 are interpolated on the calibrated curve. In this way, we get the concentration of $\mathrm{Ca}$ for every filter.

Details of the isokinetic sampling are given in Fig. 3 and by Henriquez et al. [11].

\subsection{Material}

Spheres of steel supplied by Fabio Murga S.L. (Spain) were used as filter grains in the MHEF. The size of the grains was $1 \mathrm{~mm} \pm 5 \%$. Calcium carbonate $\left(\mathrm{CaCO}_{3}\right)$ was used as dust particles. The mean diameter of these particles was determined by using a Particle size analyser of Leed \& Northrup SRA and the mean diameter was found to vary from 12 to $20 \mu \mathrm{m}$ for the effluent concentration of dust.

\subsection{Procedure}

Prior to each filtration experiment, the aerosol generator was started and allowed to run at minimum fluidization velocity until the dust particles $\left(\mathrm{CaCO}_{3}\right)$ reached a good condition of mixture with the grain of the fluidized bed (sand, $d_{\mathrm{p}}=500 \mu \mathrm{m}$ ).

After mixing for $5 \mathrm{~min}$, the dust suspension was allowed to pass through a decanting-mixing chamber. In this way, we get a determined particle size clistribution-depending on the fluidization velocity. In the decanting-mixing chamber, we mixed the dust with hot gas that enters from a blower that is connected with a heat exchanger. Therefore, we can control the particle size distribution and gas temperature at the outlet of the decanting-mixing chamber.

After that, the dust suspension was allowed to pass through the MHEF in crossflow. Dust samples were taken simultaneously at both the inlet and the exit of 


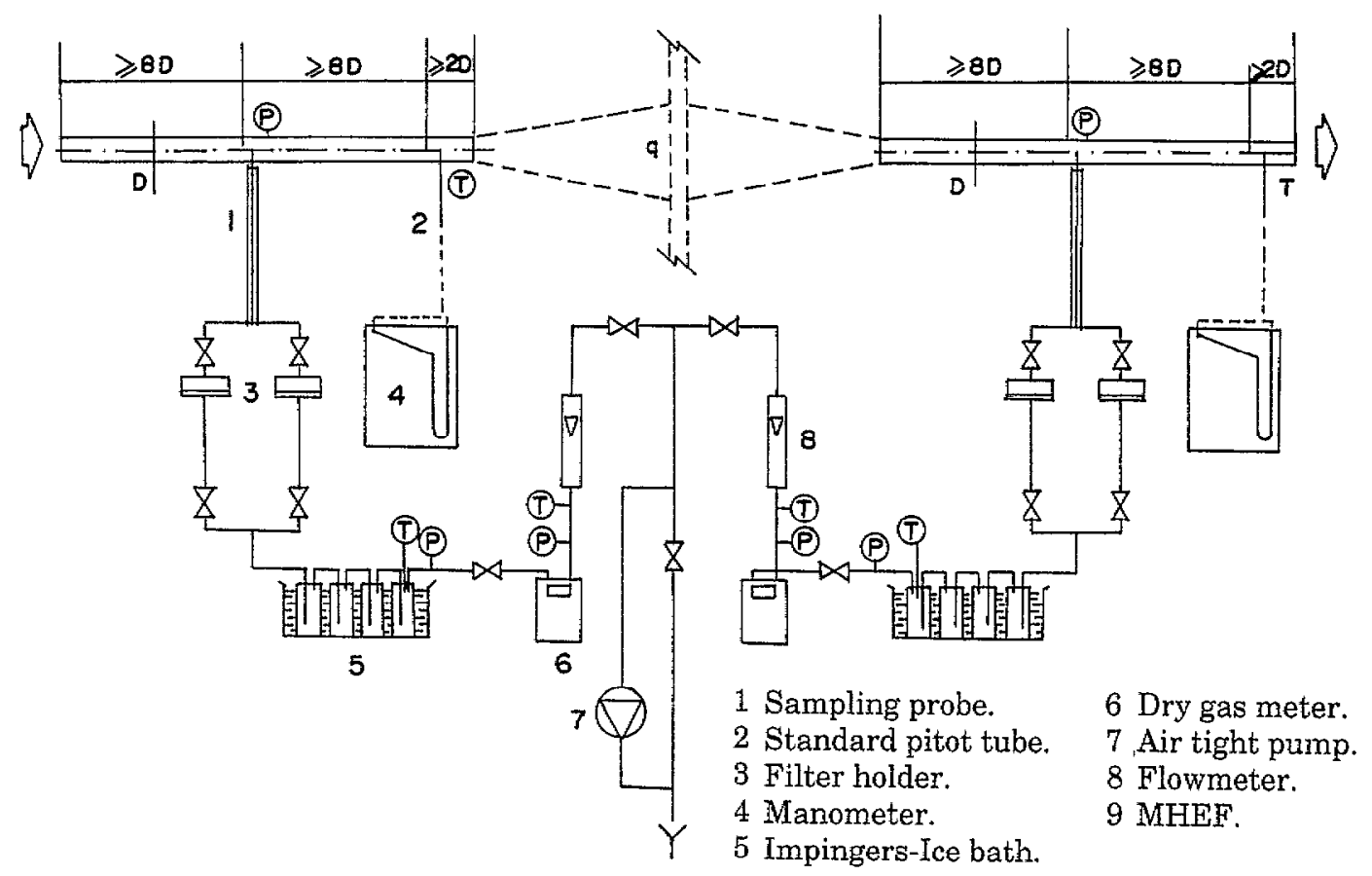

Fig. 3. Details of isokinetic sampling devices.

the MHEF at a flow rate isokinetic to that of the filter inlet. The samples were taken every $5 \mathrm{~min}$ and each experiment lasted for $\approx 60 \mathrm{~min}$.

\subsection{Experimental variables}

The experimental variables considered in this work include gas velocity, solid velocity, gas temperature, solid temperature, influent and effluent concentrations of dust and specific deposit collected by the bed, ' $m$ ' (mass of particles/bed volume).

The details of the localization of the experimental variables measured can be observed in Figs. 2 and 3. The measures of these variables have been done following the references and norms described in the EPA norms [12] and ANSI/ASME [13].

These experimental variables enable us to calculate the total collection efficiency of the filter and the thermal efficiency of heat exchange. The experimental conditions of the bulk of measurements are summarised in Table 1.

\section{Analysis and modelling}

\subsection{Heat transfer}

The two dimensional model of heat transfer-filtration used in this paper is a variation of the modelling that has recently been reported by Macias-Machin et al. [14]. The model describes the combined process of heat transfer and filtration.
The 2-D model of a crossflow heat exchanger used in this study is given by the following equations:

Fluid phase:

$\frac{\partial T}{\partial t}+u \frac{\partial T}{\partial x}=\frac{k_{\text {eff }}}{\rho_{\mathrm{f}} c_{\mathrm{f}}} \frac{\partial^{2} T}{\partial x^{2}}+\frac{h S}{\rho_{\mathrm{f}} c_{\mathrm{f}} \varepsilon}(\theta-T)$

Solid phase:

$\frac{\partial \theta}{\partial t}+v \frac{\partial \theta}{\partial y}=\frac{h S}{\rho_{s} c_{s}(1-\varepsilon)}(T-\theta)$

$\forall t=0 \quad T=\theta_{0} \quad \theta=\theta_{0}$

$\forall x=0 \quad T=T_{0} \quad \forall t$

$\forall y=0 \quad \theta=\theta_{0} \quad \forall t$

$\forall x=L \quad-k_{\mathrm{ew}} \frac{\partial T_{n, j}^{k+1}}{\partial x}=h_{\mathrm{w}}\left(T_{n, j}^{k+1}-T_{\mathrm{w}}\right)$

Table 1

Input parameters for the computational model

\begin{tabular}{ll}
\hline Bed height & $0.50 \mathrm{~m}$ \\
Bed width & $0.12 \mathrm{~m}$ \\
Cross sectional area & $0.25 \mathrm{~m}^{2}$ \\
Grain diameter & $1 \mathrm{~mm} \pm 5 \%$ \\
Bed porosity & 0.347 \\
Wall porosity & 0.56 \\
Solid velocity & $1-4 \mathrm{~cm} \mathrm{~min}^{-1}$ \\
Gas velocity & $0.42-0.475 \mathrm{~m} \mathrm{~s}^{-1}$ \\
Dust density & $2700 \mathrm{~kg} \mathrm{~m}^{-3}$ \\
Gas density & $1.2 \mathrm{~kg} \mathrm{~m}^{-3}$ \\
Specific heat of gas & $1005 \mathrm{~J} \mathrm{~kg}^{-1} \mathrm{~K}^{-1}$ \\
Specific heat of solid & $544 \mathrm{~J} \mathrm{~kg}^{-1} \mathrm{~K}^{-1}$ \\
\hline
\end{tabular}




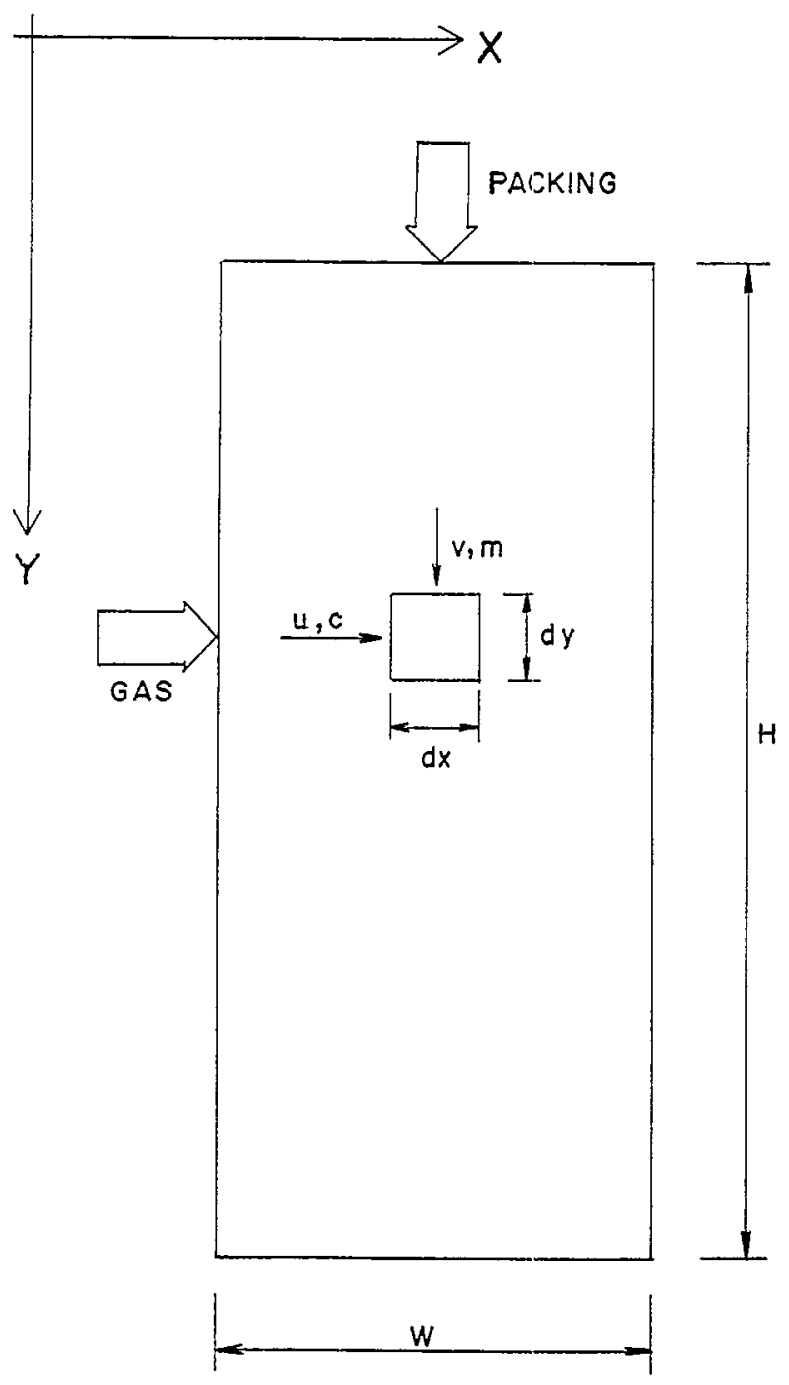

Fig. 4. Elemental $\mathrm{d} x \mathrm{~d} y$ of bed for calculating dust collection.

Eq. (3) represents the initial conditions and the Eqs. (4) and (5) are the boundary conditions that define the inlet gas and solid temperature into the MHEF. Eq. (6) represents the boundary conditions for any instant $k+$ 1 in the column $n(x=L)$ and for every file $j$.

\subsection{Filtration}

We can observe in the filtration process that the gas passes through the MHEF and the clust is collected in the medium. The dust then accumulates in the medium and is discharged together with the packing at the lower part of the filter.

The simulation of the dust collection in the bed has been done using Fig. 4 where the elemental $\mathrm{d} x \mathrm{~d} y$ is given for the dust collection process and the following equations represent this process: $v \frac{\partial m}{\partial y}+u \frac{\partial c}{\partial x}=0$

where $m$ represents the specific deposit of the dust deposited in the MHEF, $c$ is the dust concentration in the gas, $v$ is the downward velocity of the gas and $u$ is the horizontal velocity of the gas.

The particle concentration profile throughout a filter (c versus $x$ ) can often be described by the logarithmic law,

$\frac{\mathrm{d} c}{\mathrm{~d} x}=-\hat{\lambda} c$

where $\lambda$ is known as the filter coefficient and has unit of reciprocal length [15],

$\lambda=\left[\frac{6(1-\varepsilon)}{\pi}\right]^{1 / 3} \frac{\eta_{0}}{d_{\mathrm{m}}}$

where $\hat{\lambda}_{0}$ represents the single collector efficiency, $d_{\mathrm{m}}$ is the filter diameter and $\varepsilon$ is the average void ratio of the filter which is related to $m$ by the following equation:

$\varepsilon=\varepsilon_{0}-\frac{m}{\rho_{\mathrm{d}}}$

$\rho_{\mathrm{d}}$ represents density of the dust and $\varepsilon_{\mathrm{v}}$ the void fraction of the dustless medium.

Other parameters that have influence on the granular filtration are the pressure drop and the outlet dust concentration, $c$. The ability to predict accurately the progress of dust concentration and local pressure is obviously interesting from both theoretical and practical considerations. The pressure drop of the gas [16] is given by:

$-\frac{\mathrm{d} p}{\mathrm{~d} c}=\frac{1}{g}\left[\frac{150}{36} \mu_{\mathrm{f}} S^{2} \frac{(1-\varepsilon)^{2}}{\varepsilon^{3}} u+\frac{1.75}{6} \rho_{\mathrm{f}} S \frac{(1-\varepsilon)}{\varepsilon^{3}} u^{2}\right]$

The specific surface area of the medium [16], $S$, is related to that of the clean medium, $S_{\mathrm{m}}$, and of the dust, $S_{\mathrm{d}}$, as in Eq. (12):

$S=\frac{1}{1-\varepsilon}\left[\left(1-\varepsilon_{0}\right) S_{\mathrm{m}}+m \frac{S_{\mathrm{d}}}{\rho_{\mathrm{d}}}\right]$

The actual local gas velocity is given as

$u=\frac{u_{0}}{\varepsilon}$

Eqs. (1) (13) allow for simulation of the process of heat transfer and filtration in the MHEF. Therefore, we can calculate the aerosol collection in the bed and determinate the particulate solid concentration in the gas, the dust deposit in the medium, the overall collection efficiency of the MHEF and the influence of the pressure drop which is related to the local void fraction and physical properties of the gas.

The numerical solution of this set of differential equations was solved using the finite differences method for the equations of the heat transfer process. 
For the process of filtration we have used the method of Euler. After we had solved the filtration equations, the parameters obtained in the filtration's model were used to model the process of heat transfer.

\subsection{Transport properties and coefficients}

The coefficients used in the model of the MHEF are given by the following expressions:

The fluid phase effective thermal conductivities of the bed $\left(k_{\mathrm{eff}}\right)$ and in the proximity of the wall $\left(k_{\mathrm{cw}}\right)$ are given by the following expressions [17],

$k_{\text {eff }}=\varepsilon k_{\mathrm{g}}+(1-\varepsilon) k_{\mathrm{s}}\left[\frac{1}{\varphi_{\mathrm{b}} \frac{k_{\mathrm{s}}}{k_{\mathrm{g}}}+\frac{2}{3}}\right]$

and

$k_{\mathrm{ew}}=\varepsilon_{\mathrm{w}} k_{\mathrm{g}}+\left(1-\varepsilon_{\mathrm{w}}\right) k_{\mathrm{s}}\left[\frac{1}{\varphi_{\mathrm{b}} \frac{k_{\mathrm{s}}}{k_{\mathrm{g}}}+\frac{2}{3}}\right]$

where $\varphi_{b}$ represents the equivalent thickness of stagnant gas film around the contact points between particles, which aids in the transport of heat from particle to particle and $\varphi_{\mathrm{w}}$ is for contact between adjacent particles and surface of the wall [18]. $\varepsilon_{\mathrm{w}}$ is the mean void fraction of this wall layer. This parameter is calculated from Ridgway and Tarbuck [19].

The heat transfer coefficient at the wall region and containing stagnant gas as follows [20],

$h_{\mathrm{w}}^{0}=\frac{2 k_{\mathrm{ew}}}{d_{\mathrm{p}}}$

and when we have wall region with flowing gas, the heat transfer coefficient is

$h_{\mathrm{w}}=h_{\mathrm{w}}^{0}+\alpha_{\mathrm{w}} c_{\mathrm{pg}} \rho_{\mathrm{g}} u_{0}$

$\alpha_{w}$ is a constant that represents the movement and mixed of gas in the wall region [20]. A reasonable value for $\alpha_{\mathrm{w}}$ is 0.05 .

\section{Results}

The measurements obtained in the filtration experiments are the influent and effluent concentrations $c_{0}$ and $c$ expressed as $\mathrm{mg}$ of $\mathrm{CaCO}_{3}$ per $\mathrm{m}^{3}$ of air at various times. The total collection efficiency of the filter at any time, $E$, is defined as:

$E=\frac{c_{0}-c}{c_{0}}$

Normally, we can express the dynamic behaviour of the filters by the variation of ' $E$ ' with time or with respect to the mass of particles collected per bed volume ' $m$ ' (mass of particles/bed volume) [21]. The latter quantity can be evaluated as

$m=\frac{\int_{0}^{t}\left(c_{0}-c\right) u A \mathrm{~d} t}{A w}=\frac{u}{w} \int_{0}^{t}\left(c_{0}-c\right) \mathrm{d} t$

The total collection efficiency, however, does not provide a direct measurement of the particle collecting capability of the filter bed. The quantity found to be useful in characterising the particle-collecting capability of the MHEF is the so-called filter coefficient, $\lambda$ (Eq. (9)) and this is related to the unit collection efficiency, $\eta$.

Tsubaki and Tien [22] have proposed that one may assume that the unit collector efficiency of the filter grains along the gas flows in a moving bed is constant and equal to the initial unit collection efficiency, $\eta_{0}$. Since the filter grains are in motion, it becomes more difficult for deposits to form on adjoining or neighbouring filter grains.

The relationship between $\eta_{0}$ and $E$ is given in the study by Pendse and Tien [23] and this unit collection efficiency provides a meaningful indicator of the grain intrinsic ability to capture particles. For the process of heat transfer in the MHEF it is important to know the thermal efficiency, $E_{\mathrm{t}}$, as defined in [24],

$$
\begin{aligned}
E_{\mathrm{t}} & =\frac{\text { Heat taken by cold solids in time ' } t \text { ' }}{\text { Maximum possible take up in time ' } t \text { ' }} \\
& =\frac{\text { Heat lost by hot gas in time ' } t '}{\text { Maximum possible heat lost in time ' } t \text { ' }} \\
E_{\mathrm{t}} & =\frac{\Delta T_{\mathrm{h}}}{\Delta T_{\max }}=\left[\frac{T_{\mathrm{h}, \text { in }}-\bar{T}_{\mathrm{h}, \text { out }}}{T_{\mathrm{h}, \mathrm{in}}-T_{\mathrm{c}}}\right]_{\text {gas }}
\end{aligned}
$$

Assuming that heat losses to surroundings are negligible and that the mean physical properties independent of temperature can be used. Also, assuming constant mass flow rate and gas inlet temperature.

\subsection{Filtration in the MHEF}

The results shown in Fig. 5 were obtained at two gas velocities $u=0.475 \mathrm{~m} \mathrm{~s}^{-1}$ and $u=0.521 \mathrm{~m} \mathrm{~s}^{-1}$ and $\mathrm{a}$ fixed solid velocity of $v=1 \mathrm{~cm} \mathrm{~min}{ }^{-1}$. These experiments were realised at ambient temperature $\left(20^{\circ} \mathrm{C}\right)$ and at efficiencies of filtration between 88 and $99 \%$. The data plotted for this solid velocity show that the filtration efficiency increases with the increase of gas flow.

The effect of solid velocity on particle collection was found to be important (as shown in Fig. 6). In Fig. 6 the total collection efficiency of the filter is given as a function of time. The data shown in this figure was obtained for the same gas velocity $\left(u=0.521 \mathrm{~m} \mathrm{~s}^{-1}\right)$ and we have varied the solid velocity in the range $1-4.2$ $\mathrm{cm} \mathrm{min}^{-1}$. The total collection efficiency calculated 


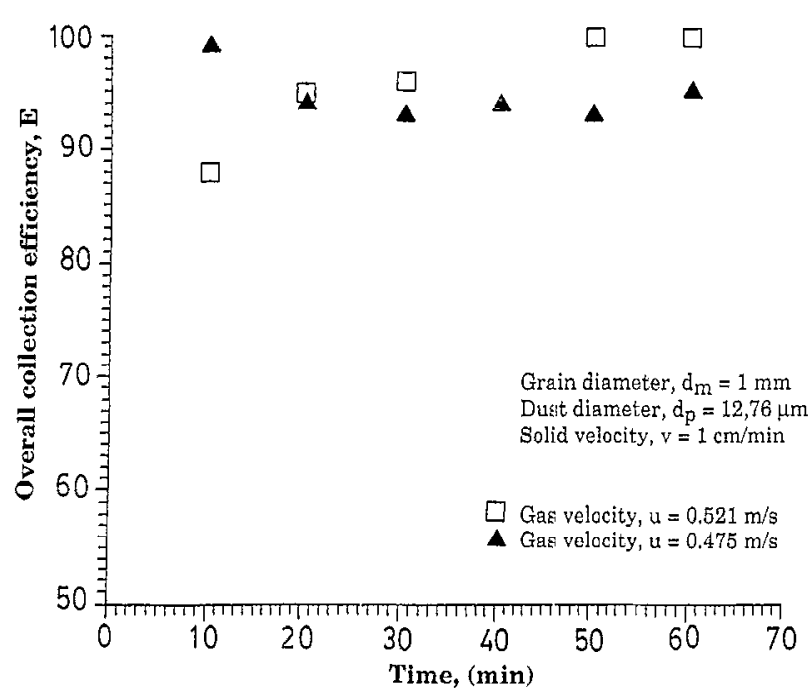

Fig. 5. Total collection efficiency at ambient temperature $\left(T=20^{\circ} \mathrm{C}\right)$.

decreases when the solid velocity increases. The total collection efficiency observed for the case $v=4.2 \mathrm{~cm}$ $\min ^{-1}$ was lower than the total collection efficiency for the case $v=1 \mathrm{~cm} \mathrm{~min}^{-1}$.

The effect of solid velocity on the process of particle collection is very important and affects the process of heat transfer of the MHEF, too. [25]. This situation provides a good indication of the significant influence of gas velocity in the process on heat transfer-filtration because if we increase the solid velocity the total dust collection efficiency decreases significantly but the thermal efficiency of the MHEF increases.

The influence of the temperature on the total collection efficiency, ' $E$ ', of the MHEF has been plotted as a function of time in Fig. 7. We have used a dust of $\mathrm{CaCO}_{3}$ with a mean diameter particle of $12 \mu \mathrm{m}$ where the size range varied between 5 and $50 \mu \mathrm{m}$. The grade efficiency decreases when temperature increases, in agreement with filtration theory. Since the viscosity ' $\mu$ '

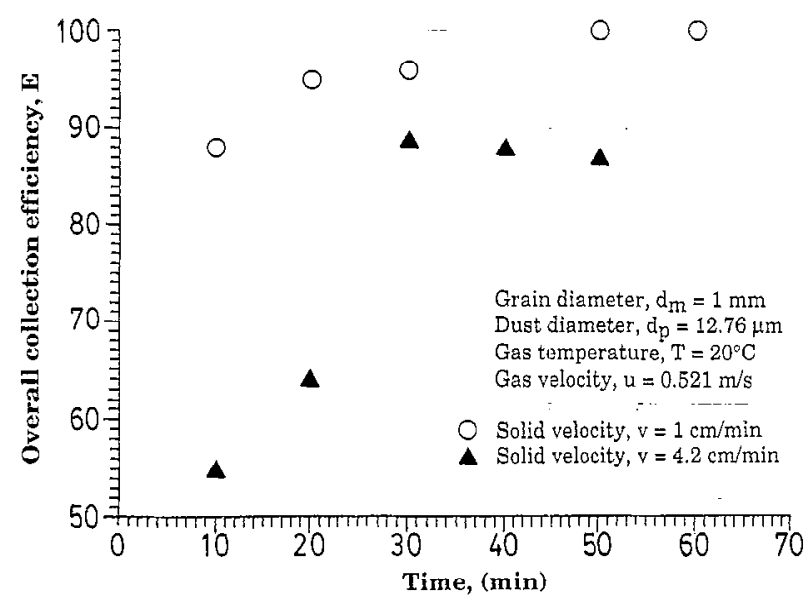

Fig. 6. Effect of solid velocity on total collection efficiency.

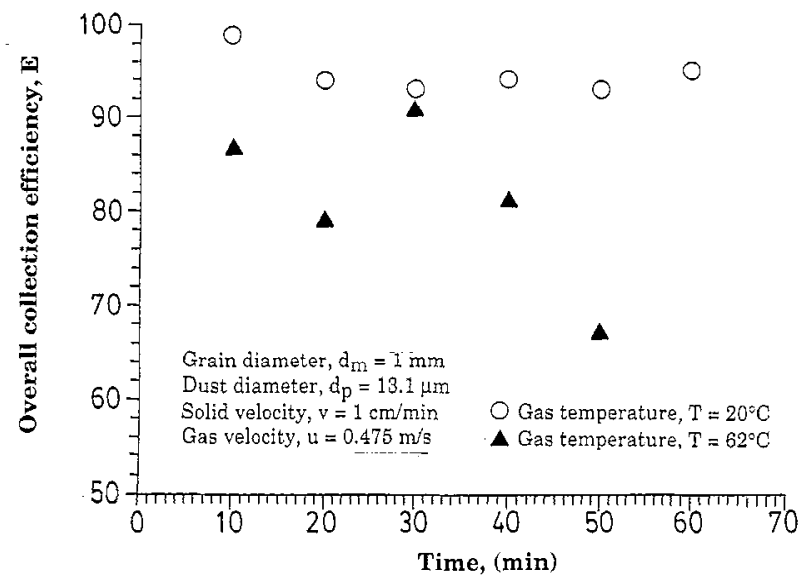

Fig. 7. Effect on gas temperature on total collection efficiency.

of the gas increases with increasing temperature and because the Cunningham factor slip correction for particles larger than $1 \mu \mathrm{m}$ can be approximated to $\mathrm{Cu}=1$ [26], the collection efficiency decreases when the temperature increases due to decreasing values of the inertial parameter (Stokes Number).

A comparison between separation efficiency measurements using the same gas and solid velocities for different gas temperatures $\left(20\right.$ and $62^{\circ} \mathrm{C}$ ) is shown in Fig. 7 . We can observe that the collection efficiency decreases when temperature increases. Despite the fact that the grain size $\left(d_{\mathrm{p}}=1 \mathrm{~mm}\right)$ is relatively coarse and the superficial velocity is comparatively high, this total collection efficiency could be increased if we decrease the grain size $(\leq 1 \mathrm{~mm})$ and used lower solid velocity. These possibilities allow to improve the total collection efficiency of the MHEF or to enlarge the filter depth because ' $E$ ' is strongly influenced by it.

\subsection{Heat transfer in the MHEF}

Results of the 2-D model of filtration and heat exchange are compared with experimental measurements from different operation conditions. The experimental bed provides detailed transient temperature distributions during the process of filtration and heat exchange.

Fig. 8 compares the fluid temperature at the centre of the MHEF with the prediction of the model. The results demonstrate favourable agreement between the experimental data and predicted numerical values. The best agreement obtained for the model is when axial thermal dispersion is decreased by using a higher value of the heat transfer coefficient. Numerical experiments with the model indicate that over a reasonable range of values, $k_{\text {eff }}$, has a negligible effect on the shape of the temperature curves for the process of heat transferfiltration. 


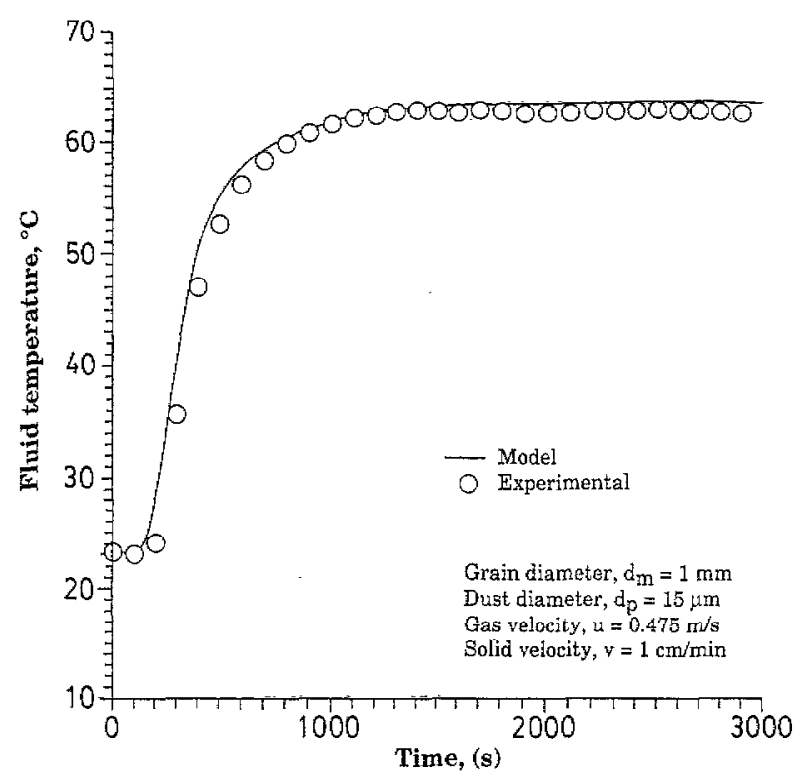

Fig. 8. Measured and computed fluid temperature response of the MHEF.

Solid-fluid temperature measurements during transient operation allow to determine local values of ' $h$ ' in various locations. The resulting average local values of ' $h$ ' correspond to values of the Nusselt number lower than those of Ranz [27] (fixed bed) and higher than those of Turton and Levenspiel [28] and MaciasMachin et al. [29] (fluidized bed). It can be observed that the heat transfer coefficients obtained varied from 344 to $352 \mathrm{~W} \mathrm{~m}^{-2} \mathrm{~K}^{-1}$, for a solid velocity of $1 \mathrm{~cm}$ $\mathrm{min}^{-1}$ and a gas velocity from 0.420 to $0.521 \mathrm{~m} \mathrm{~s}^{-1}$. The mean diameter of the dust used varies from 12.76 to $13.1 \mu \mathrm{m}$.

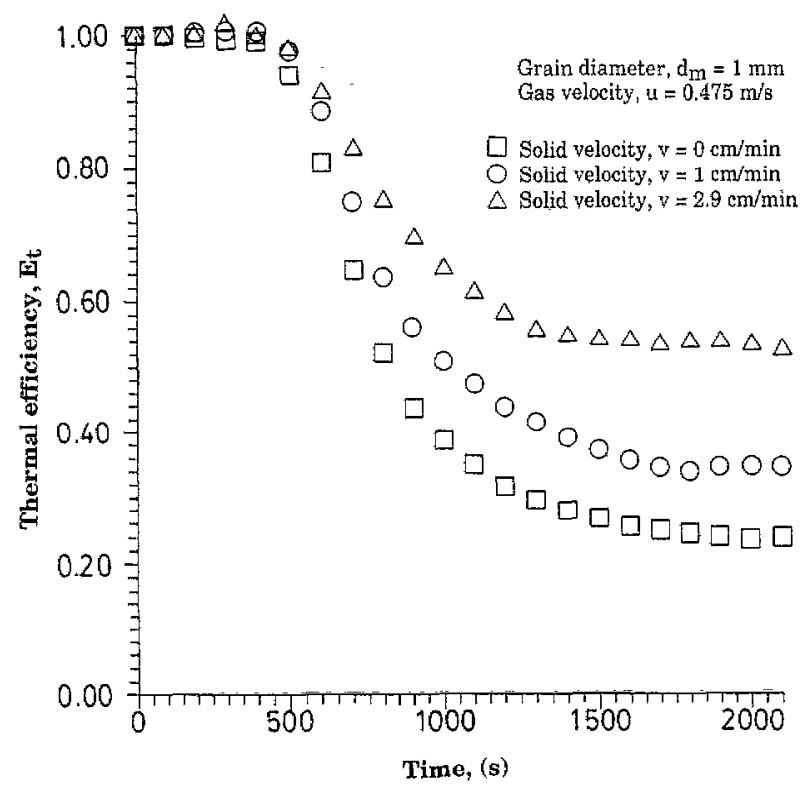

Fig. 9. Thermal efficiency of the MHEF at various solid velocities.
Fig. 9 shows the thermal efficiency for a gas velocity $0.475 \mathrm{~m} \mathrm{~s}^{-1}$ for different solid velocities $v=0$ (fixed bed) and $v=1-2.9 \mathrm{~cm} \min ^{-1}$ (moving bed). We can observe that for the solid velocity $v=0$ (fixed bed) a minimum and a maximum thermal efficiency occurs for a moving bed with higher solid velocity $\left(2.9 \mathrm{~cm} \mathrm{~min}^{-1}\right)$. When increasing the solid velocity, the total collection efficiency, ' $E$ ', decreases but thermal efficiency increases ' $E_{\mathrm{c}}$ '. Therefore, it is necessary to optimise the experimental variables and operation mode of combined filtration heat exchange to get higher total collection efficiency, ' $E$ ', or higher thermal efficiency, ' $E$ ', or both.

\section{Conclusions}

This study identifies the effects of gas and solid velocities, particle size distribution of the dust, flow distribution on the dynamic response of a moving bed heat cxchangcr-filter, subjccted to an arbitrary time varying inlet fluid temperature, for both processes of flitration and heat exchange.

The effect of solid velocity on particle collection is very important and the total collection efficiency decreases when the solid velocity increases. But, if we take into account the process of heat transfer when we increase the solid velocity the thermal efficiency increases.

Measured values of total collection efficiency in different experiments indicate that ' $E$ ' decreases when increasing temperature and this question is in agreement with the filtration theory.

A predictive numerical model is developed which incorporates variations in void fraction, velocity and transport coefficients due to combined process of heat exchange-filtration.

The model is capable of predicting the 2-D transient behaviour of the MHEF for various solid velocities. The model is verified by comparison with experimental data from combined processes of heat exchange-filtration.

\section{Nomenclature}

$c_{0} \quad$ influent dust concentration in the gas $\left(\mathrm{kg} \mathrm{m}^{-3}\right)$

$c$ effluent dust concentration in the gas $\left(\mathrm{kg} \mathrm{m}^{-3}\right)$

$c_{\mathrm{f}} \quad$ specific heat of gas $\left(\mathrm{J} \mathrm{kg}^{-1} \mathrm{~K}^{-1}\right)$

$c_{\mathrm{s}} \quad$ specific heat of solid $\left(\mathrm{J} \mathrm{kg}^{-1} \mathrm{~K}^{-1}\right)$

$d_{\mathrm{m}} \quad$ grain diameter $(\mathrm{m})$

$E$ overall collection efficiency (-)

$H$ height of the filter (m)

$h$ heat transfer coefficient of the bed $\left(\mathrm{W} \mathrm{m}^{-2}\right.$ $\mathrm{K}^{-1}$ ) 
$h_{\mathrm{w}} \quad$ heat transfer coefficient in the wall region of the bed ( $\mathrm{W} \mathrm{m}^{2} \mathrm{~K}^{-1}$ )

$k_{\text {eff }}$ effective thermal conductivity of a moving bed ( $\mathrm{W} \mathrm{m}^{-1} \mathrm{~K}^{-1}$ )

$k_{\mathrm{w}}$ effective thermal conductivity of a thin layer of bed near the wall surface $\left(\mathrm{W} \mathrm{m}^{-1} \mathrm{~K}^{-1}\right)$

$m$ dust deposition in the medium $\left(\mathrm{kg} \mathrm{m}^{-3}\right)$

$S \quad$ specific surface area of the medium $\left(\mathrm{m}^{2} \mathrm{~m}^{-3}\right)$

$T \quad$ fluid temperature (K)

$t$ time (s)

$u \quad$ gas velocity $\left(\mathrm{m} \mathrm{s}^{-1}\right)$

$u_{0} \quad$ superficial gas velocity $\left(\mathrm{m} \mathrm{s}^{-1}\right)$

$v$ solid velocity $\left(\mathrm{m} \mathrm{s}^{-1}\right)$

$w$ maximum width of the MHEF (m)

Greek symbols

$\eta \quad$ collection efficiency of a single sphere (-)

$\varepsilon \quad$ void fraction $(-)$

$\rho_{\mathrm{f}} \quad$ gas density $\left(\mathrm{kg} \mathrm{m}^{-3}\right)$

$\rho_{\mathrm{s}} \quad$ solid density $\left(\mathrm{kg} \mathrm{m}^{-3}\right)$

$\mu \quad$ viscosity $\left(\mathrm{kg} \mathrm{m}^{-1} \mathrm{~s}^{-1}\right)$

$\theta \quad$ solid temperature $(\mathrm{K})$

$\begin{array}{ll}\text { Subscripts } \\ \mathrm{d} & \text { dust } \\ \text { eff } & \text { effective } \\ \mathrm{f} & \text { fluid } \\ \mathrm{m} & \text { medium } \\ \mathrm{0} & \text { initial } \\ \mathrm{w} & \text { wall }\end{array}$

\section{Acknowledgements}

The financial support from CICYT of the Spanish Government is appreciated (Project number AMB921278).

\section{References}

[1] W. Licht, Air Pollution Control Engineering, Chapter 8, Marcel Dekker Inc., New York, 1988.

[2] E. Keigel, Method and apparatus for heat and mass transfer between a flowing medium and fine solid particles, German Patent Offen, DE 3639046 A1, 1988.

[3] O. Levenspiel, Countercurrent heat exchanger for two streams of solid using heat pipes, US Patent No. 4.4.08.653, 1983.

[4] G. Meumier, Apparatus for heat exchange between solid particles and gaseous stream, European Patent, RP 9.455, 1980.

[5] O. Schumacher, Energy or material transfer between small particles and a fluid, Patent Germany Offen, DE 3504214 A1, 1986.

[6] E. Jaraiz, A. Estevez, J. Cuellar, A. Macias-Machin, Spanish Patent No. 9.102.728, 1991.
[7] A. Macias-Machin, A. Estevez, J. Cuellar, E. Jaraiz, Simple design of a crossfiow Moving Bed Heat Exchanger-Filter (MHEF), Filtration and Separation 29 (1991) 155-161.

[8] A. Macias-Machin, G. Winter, G. Montero, S. Garcia, Modelling of a new Heat Exchanger-Filter for cleaning dusty gases, Proc. 6th World Filtration Congress, Nagoya, Japan, 1993, pp. $790-793$.

[9] V. Henriquez, A. Lozano, A. Macias-Machin, Influence of a decanting-mixing chamber in aerosol generation, Proc. Int. Symp. Filtration and Separation, Salamanca, Spain, 1995, pp. $98-106$.

[10] V. Henriquez, Filtración y recuperación de gases calientes contaminantes, $\mathrm{PhD}$ Thesis, University of Las Palmas de Gran Canaria, Spain, 1996.

[11] V. Henriquez, A. Lozano, A. Macias-Machin, Gas Filtration in a Heat Exchanger-Filter MHEF. Preliminary studies, Proc. International Symposium on Filtration and Separation, Salamanca, Spain, 1995, pp. 166-173.

[12] EPA Norms, Stationary Source sampling methods, Method 1-A, August 18th, 1977.

[13] ANSI Norms, American Society of Mechanical Engineers (Eds.), New York, 1980.

[14] A. Lozano, V. Henriquez, A. Macias-Machin, Modelling of a new crossflow moving-bed heat exchanger-filter (MHEF), Filtration and Separation 33 (1996) 69-74.

[15] C. Tien, Granular filtration of aerosol and hydrosol, Chapter 6 , Butterworth, Stoneham, MA, 1989.

[16] S. Mizukami, M. Wakabayashi, H. Murata, Interaction between pressure drop of gas and flow of medium in a moving granular bed filter, Part. Sci. Technol. 5 (1987) 131-142.

[17] D. Kunii, O. Levenspiel, A general equation for the heat transfer coefficient at wall surfaces of gas/solid contactors, Ind. Eng. Chem. Res. 30 (1991) 136-141.

[18] S. Yagi, D. Kunii, Studies of heat transfer near wall surface in packed bed, AICHE J. 6 (1960) 97-104.

[19] K. Ridgway, K.J, Tarbuck, Voidage fluctuations in randomlypacked beds of spheres adjacent to a containing wall, Chem. Eng. Sci. 23 (1968) 1147-1155.

[20] D. Kunii, O. Levenspiel (Eds.), Fluidization Engineering, Butterworth-Heinemann, Stoneham, MA, 1991, p. 326.

[21] H.W. Chiang, C. Tien, Dynamics of deep-bed filtration, AICHE J. 31 (1985) 1360-1371.

[22] J. Tsubaki, C. Tien, Gas filtration in granular moving beds. An experimental study, Can. J. Chem. Eng. 66 (1988) 271-275.

[23] H. Pendse, C. Tien, General correlation of the initial collection efficiency of granular filter beds, AICHE J. 28 (1982) 677-686.

[24] O. Levenspiel, Engineering Flow and Heat Exchange, Plenum Press, New York, 1984, p. 306.

[25] V. Henriquez, A. Lozano, D. Santana, A. Macias-Machin, Heat Exchange-Filtration of Hot Gases: Effect of the operating conditions, Proc. 7th World Filtration Congress, Budapest, Hungary, 1996, pp. $738-742$.

[26] W. Peukert, F. Löffler, Influence of temperature on particle separation in granular bed filters, Powder Technol. 68 (1991) $263-270$.

[27] W.E. Ranz, Chem. Eng. Progr. 48 (1952) 247.

[28] R. Turton, O. Levenspiel, An experimental method to determine the heat transfer coefficient between fine fluidized particles and air via changes in magnetic properties, Int. J. Heat Mass Transf. $\overline{3} 2$ (1989) 289-296.

[29] A. Macias-Machin, L. Oufer, N. Wannenmacher, Heat transfer between an inmersed wire and a liquid fluidized bed, Powder Technol. 66 (1991) 281-284. 